\title{
Karyotype and DNA-Methylation Responses in Myelodysplastic Syndromes following Treatment with Traditional Chinese Formula Containing Arsenic
}

\author{
Sun Shuzhen, Ma Rou, Hu Xiaomei, Yang Xiao-hong, Xu Yong-gang, \\ Wang Hongzhi, and Yang Xiu-Peng \\ National Hematological Medical Center of Traditional Chinese Medicine, Department of Hematology, Xiyuan Hospital, \\ China Academy of Chinese Medical Sciences, Beijing 100091, China \\ Correspondence should be addressed to Ma Rou, xyxysys@yahoo.com.cn
}

Received 30 July 2012; Revised 3 September 2012; Accepted 10 September 2012

Academic Editor: Shao Li

Copyright (C) 2012 Sun Shuzhen et al. This is an open access article distributed under the Creative Commons Attribution License, which permits unrestricted use, distribution, and reproduction in any medium, provided the original work is properly cited.

\begin{abstract}
We have previously shown that arsenic-containing Chinese herbal formula, Qing-Huang powder capsule (containing tetraarsenic tetrasulfide, $\mathrm{As}_{4} \mathrm{~S}_{4}$ ), is effective in the treatment of myelodysplastic syndrome (MDS); yet the underlined mechanisms remain unclear. In this study, using standard cytogenetic analysis (G-banded) and global DNA methylation method (ChIP-on-chip assays), we aimed to determine the effect of arsenic-containing Chinese herbal formula on karyotype status and the genomic methylation level in primarily diagnosed MDS patients. Correlation of aberrant DNA methylation and chromosome aberrations in MDS was also investigated. We found that the number of genes with aberrant DNA methylation was highest in MDS patients with normal karyotype, followed by trisomy 8 karyotype, and relatively low in patients with cytogenetic abnormalities other than trisomy 8 . Treatment with arsenic-containing Chinese herbal formula had no effects on karyotype status, but resulted in a significant genomewide demethylation. Our research uncovered a DNA demethylating activity of arsenic-containing Chinese herbal formula in the treatment of MDS.
\end{abstract}

\section{Introduction}

Myelodysplastic syndromes (MDS) are characterized by functional abnormalities of hematopoietic lineages. Due to ineffective hematopoiesis, patients with MDS present with cytopenia(s) associated with morphological dysplasia and/or increase in number of blasts, and can progress to acute myeloid leukemia. The laboratory diagnostic strategy in MDS has evolved significantly over the years. Currently the integrated cytogenetic/genetic approach is critical for diagnosis and prognosis of MDS. DNA methylation is a biochemical process that is important for normal development in higher organisms. It involves the addition of a methyl group to the 5 position of the cytosine pyrimidine ring carried out by DNA methyltransferases. It has been shown that MDS patients, especially high-risk MDS, have abnormal hypermethylation of tumor suppressor genes [1].
Our previous works indicated that Chinese herbal formula Qing-Huang powder (containing Realgar $\mathrm{As}_{4} \mathrm{~S}_{4}$ ) is effective in the treatment of myelodysplastic syndrome (MDS) [2-4]. In the year 2011, we reported that of 124 cases of MDS patients treated with arsenic-containing herbal formula, complete remission and partial remission rate was achieved in 29 (23.4\%) patients; hematologic improvement was achieved in $61(49.2 \%)$ patients. The total efficacy rate was $80.6 \%$. The clinical outcome after treatment was closely correlated with WHO Classification of myelodysplastic syndromes, MDS-risk scores, "International Prognostic Scoring System" (IPSS), and cyogenetic abnormality. However the underlined mechanisms remain unclear. To address the value of traditional medicine, efforts are required to delineate how the clinically effective formulae work at the molecular, cellular, and organism levels. 
In this paper, using standard cytogenetic analysis (Gbanded) and global DNA methylation method (ChIP-onchip assays), we aimed to determine the effect of arseniccontaining Chinese herbal formula on karyotype status, the genomic methylation level as well as the correlation between aberrant DNA methylation and chromosome aberrations.

\section{Materials and Methods}

2.1. Patients and Treatment Regimen. Twenty-five patients enrolled in this study were from outpatient clinics between 2009 and 2011 after giving informed consent. All patients were diagnosed according to WHO classification system (2008) and international prognostic scoring system (IPSS) [5]. The inclusion criteria: measurements of clinical response were defined according to standardized response criteria for myelodysplastic syndromes established by the International Working Group [6,7]; patient had to be 18 to 75 years old; patients must not have received another investigational or approved therapy for MDS within 4 weeks of study enrollment; patients had no history of severe dysfunction of heart, liver, or kidney; all patients provided informed consent before this study. The exclusion criteria: patients had severe adverse effects during treatment; patients were allergic to tested drugs; patients were pregnant, breast-feeding, or had a mental disorder during treatment.

All patients orally took arsenic-containing Qing-Huang powder capsule combined with Bupi Yishen Decoction for 6 months. Qing-Huang powder capsule $0.4 \mathrm{~g} /$ day (containing Indigo $0.24 \mathrm{~g}$, and Realgar $0.16 \mathrm{~g}$ ); Bupi Yishen Decoction once a day, consisting of Sheng-shu-di, Shan-yao, Shan-yurou, Dan-pi, Fu-ling, Ze-xie, Bu-gu-zhi, Tu-si-zi, Zhi-shouwu, Tai-zi-shen, Bai-zhu, Sheng-jiang, Da-zao, Sang-ren, $\mathrm{Nu}$-zhen-zi, and Han-lian-cao were added for the patient with symptoms of Yin deficiency; Suo-yang, Ba-ji-tian, Guzhi, Zhi-fu-pian were added for the patient with symptoms of Yang deficiency.

2.2. Detection of Blood Arsenic Concentration. After treatment with Qing-Huang powder capsule, arsenic level in peripheral blood was detected by the coupling of high performance liquid chromatography (HPLC) with inductively coupled plasma mass spectrometry (ICP-MS) with the lowest detection limitation of $0.03 \mathrm{ug} / \mathrm{L}$.

2.3. Cytogenetic Analysis. Cytogenetic analysis was performed on marrow aspirates according to the standard methods. Chromosome preparations were G-banded using trypsin and Giemsa, and karyotypes were described according to International System for Human Cytogenetic Nomeclature (ICSN) [8]. Major aberrant clone was confirmed by fluorescence in situ hybridization (FISH), probes including CEP8, D5S23-5q31, D7S522-CEP7, and D20S108.

2.4. DNA Methylation and Data Analysis. Methylation detection was performed using Affymetrix USA GeneChip Human Promoter 1.0 Array according to the manufacturer's protocol (detailed protocol can be found at http://www .affymetrix.com/). Briefly, bone marrow mononuclear cells from MDS patients were collected before and after treatment. Methylated fraction of DNA was obtained using EpiQuik Methylated DNA Immunoprecipitation Kit (Promega). DNA target was PCR-amplified, fragmented, and terminally labeled. After hybridization, washing, and staining, the chip was scanned following the instructions. Conditions of chips before and after standardization were shown in supplemental Figures 1 and 2 (see Supplementary Material available online at doi:10.1155/2012/969476).

DNA methylation detection was achieved in all 25 patients before treatment, in 14 patients after treatment. DNA methylation information from 5 normal cases traced from NCBI public data Bank served as control <http:// www.ncbi.nlm.nih.gov/>. Patient characteristics are listed in Table 1.

2.5. Data Analysis and Statistics. All data were analyzed using two tools-Gene Ontology (GO) analysis and Pathway analysis-from Gene Ontology database and the KEGG database, respectively.

Gene Ontology online database was established by the Gene Ontology Consortium in 2000. The Gene Ontology project is a major bioinformatics initiative with the aim of standardizing the representation of gene and gene product attributes across species and databases. The database can be used to search or predict disease-related aberrantly expressed gene/function $[9,10]$. The KEGG database has been in development by Kanehisa Laboratories since 1995. KEGG is a collection of online databases dealing with genomes, enzymatic pathways, and biological chemicals. KEGG connects known information on molecular interaction networks, such as pathways and complexes (this is the Pathway Database), information about genes and proteins generated by genome projects (including the gene database), and information about biochemical compounds and reactions (including compound and reaction databases). The higher order functional information is stored in the Pathway Database, which contains graphical representations of cellular processes, such as metabolism, membrane transport, signal transduction, and cell cycle [11-15].

GO analysis and Pathway analysis are two major tools based on the above two bioinformatics databases and are frequently used to analyze gene and gene function via gene Chip assay. GO analysis is more specific about single gene function, while Pathway analysis is more about networks of gene interactions in the cells, and integrating the two tools will yield better results on gene function analysis.

In this study, we used both GO analysis (based on Gene Ontology database) and Pathway analysis (based on KEGG database) to map the corresponding genomic position and function. Statistical significance of the difference between values of methylation levels for different samples/groups were assessed using Fisher and multiple comparison test with $P<0.05$, false discovery rate (FDR) $<0.05$ as filtering cut-off point. 


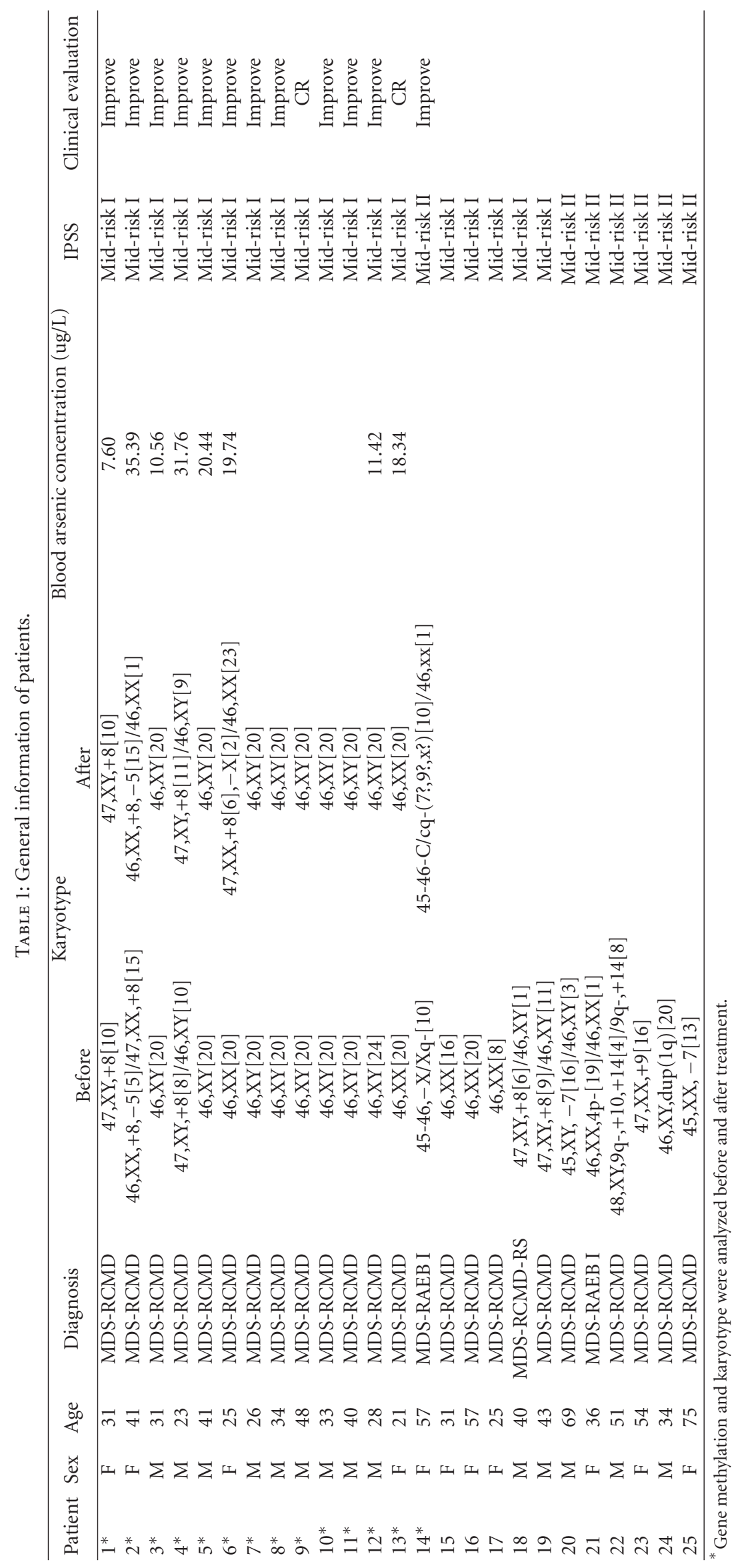


TABLE 2: Functions of genes with hypermethylation.

\begin{tabular}{|c|c|c|c|c|}
\hline Go_id & Go_name & Diffgene_count & $P$ value & FDR \\
\hline GO:0050896 & Response to stimulus & 51 & $3.16 E-22$ & $2.73 E-19$ \\
\hline GO:0006355 & Regulation of transcription, DNA-dependent & 141 & $7.10 E-17$ & $3.07 E-14$ \\
\hline GO:0007275 & Multicellular organismal development & 105 & $2.50 E-16$ & $7.23 E-14$ \\
\hline GO:0045893 & Positive regulation of transcription, DNA-dependent & 59 & $7.24 E-11$ & $1.57 E-08$ \\
\hline GO:0007165 & Signal transduction & 115 & $1.58 E-10$ & $2.73 E-08$ \\
\hline GO:0000278 & Mitotic cell cycle & 37 & $5.86 E-08$ & $7.84 E-06$ \\
\hline GO:0006811 & Ion transport & 60 & $7.95 E-08$ & $9.83 E-06$ \\
\hline GO:0006915 & Apoptosis & 61 & $2.62 E-07$ & $2.54 E-05$ \\
\hline GO:0007264 & Small GTPase mediated signal transduction & 38 & $2.69 E-07$ & $2.59 E-05$ \\
\hline GO:0006810 & Transport & 59 & $4.52 E-07$ & $3.58 E-05$ \\
\hline GO:0006357 & Regulation of transcription from RNA polymerase II promoter & 31 & $4.58 E-07$ & $3.61 E-05$ \\
\hline GO:0016568 & Chromatin modification & 26 & $5.63 E-07$ & $4.06 E-05$ \\
\hline GO:0007049 & Cell cycle & 42 & $6.84 E-07$ & $4.55 E-05$ \\
\hline GO:0000122 & Negative regulation of transcription from RNA polymerase II promoter & 44 & $1.50 E-06$ & $9.12 E-05$ \\
\hline GO:0006281 & DNA repair & 33 & $2.47 E-06$ & $1.36 E-04$ \\
\hline
\end{tabular}

This table shows parts of 156 category functions of genes with hypermethylation in 25 MDS patients. go_id: gene ID number in Gene Ontology database; go_name: category of function of gene; diffgene_count: number of gene methylated; $P$ value: $P<0.01$ indicating methylation is significant; FDR: false discovery rate, $P<0.05$ as filter cutoff rate.

TABLE 3: Pathways of hypermethylated genes.

\begin{tabular}{|c|c|c|c|c|}
\hline Path_id & Path_name & Diffgene_count & $P$ value & FDR \\
\hline 5200 & Pathways in cancer & 41 & $2.22 E-05$ & $1.15 E-03$ \\
\hline 5120 & Epithelial cell signaling in Helicobacter pylori infection & 14 & $1.11 E-04$ & $2.86 E-03$ \\
\hline 3050 & Proteasome & 10 & $5.40 E-04$ & $7.31 E-03$ \\
\hline 4144 & Endocytosis & 26 & $5.83 E-04$ & $7.53 E-03$ \\
\hline 4062 & Chemokine signaling pathway & 24 & $1.20 E-03$ & $9.49 E-03$ \\
\hline 4666 & Fc gamma R-mediated phagocytosis & 15 & $1.48 E-03$ & $9.98 E-03$ \\
\hline 4650 & Natural killer cell mediated cytotoxicity & 19 & $1.75 E-03$ & $1.03 E-02$ \\
\hline 5211 & Renal cell carcinoma & 12 & $2.13 E-03$ & $1.07 E-02$ \\
\hline 5210 & Colorectal cancer & 11 & $2.54 E-03$ & $1.09 E-02$ \\
\hline 4670 & Leukocyte transendothelial migration & 16 & $3.92 E-03$ & $1.40 E-02$ \\
\hline 4612 & Antigen processing and presentation & 12 & $4.43 E-03$ & $1.49 E-02$ \\
\hline 4360 & Axon guidance & 17 & $4.74 E-03$ & $1.54 E-02$ \\
\hline 4012 & ErbB signaling pathway & 13 & $4.91 E-03$ & $1.57 E-02$ \\
\hline 310 & Lysine degradation & 9 & $5.37 E-03$ & $1.63 E-02$ \\
\hline 4740 & Olfactory transduction & 38 & $6.37 E-03$ & $1.80 E-02$ \\
\hline
\end{tabular}

This table shows parts of 46 pathways of genes with hypermethylation in 25 MDS patients. Path_id: gene ID number in KEGG database; Path_name: pathway of gene; diffgene_count: number of gene methylated in specific pathway; $P$ value: $P<0.01$ indicating methylation is significant; FDR: false discovery rate, $P<$ 0.05 as filter cutoff rate.

\section{Results}

3.1. DNA Methylation before Treatment. According to conclusion drawn from GO analysis, 1063 hypermethylated genes were identified in 25 MDS patients compared with normal control, which consisted of $2.7 \%$ of the 4 million genes being tested. The hypermethylated genes involve 156 functional pathways, including response to stimulus, regulation of transcription DNA-dependent, multicellular organismal development, DNA-dependent positive regulation of transcription, signal transduction, apoptosis, cell cycle, and DNA repair, and so forth, as listed in Table 2 (parts of 156 functional pathways).

Using Pathway analysis, we observed that 318 hypermethylated genes were identified in $25 \mathrm{MDS}$ patients compared with control. The functions of these genes involve 46 different pathways, including pathways in cancer, Endocytosis, chemokine signaling pathway, ErbB signaling pathway, olfactory transduction, and so forth, as listed in Table 3 (parts of 46 functional pathways).

Consistent with multiple reports [16-23] and online database (http://www.ncbi.nlm.nih.gov/gene), our results 


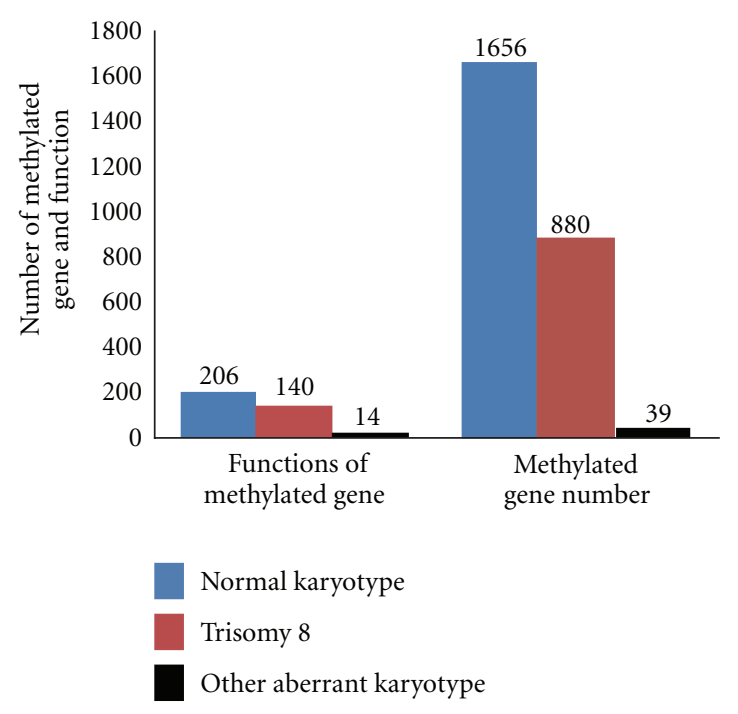

(a)

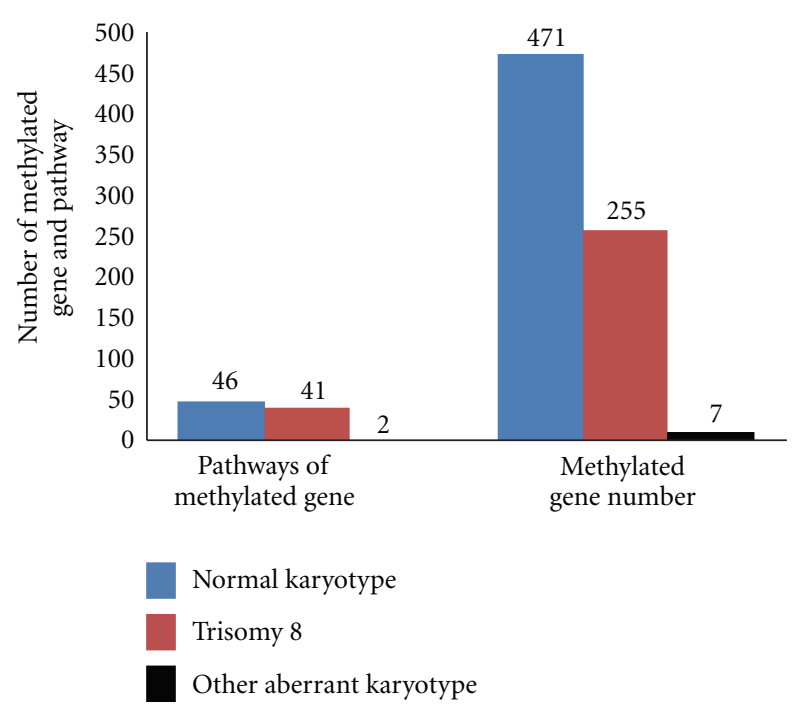

(b)

FIGURE 1

indicate that MDS patients have aberrant DNA hypermethylation. Specifically, we confirmed that the following 27 genes, involving transcription control, signaling transduction, cell development, and adhesion, as well as cancer, are hypermethylated in MDS patients: ARNT2, E2F3, HDAC1, PIAS3, TCF7L1, TGFBR1, DCC, EPAS1, MET, WNT16, WNT6, WNT8B, FZD1, NCOA4, NFKB1, BCR, FASLG, FLT3LG, IGF1R, KIT, MAP2K1, PIK3R2, PLCG1, PLCG2, RALBP1, TRAF3, and SRC.

3.2. Karyotype and Gene Methylation. Among 25 MDS patients, normal karyotypes were observed in 12 cases, trisomy +8 was observed in 6 cases. The remaining 7 patients were cases of other type of aberrant karyotype, including 2 cases of -7 , one case of $4 \mathrm{p}-,+9$, dup(1q), $-\mathrm{X} / \mathrm{Xq}-$, and complex karyotype, respectively (Table 1 ).

Compared to healthy control, as analyzed by GO analysis, the number of aberrant DNA methylation is higher in patients with normal karyotype (1656 counts) and trisomy 8 karyotype ( 880 counts). DNA methylation is relative low in patients with cytogenetic abnormalities other than trisomy 8 (39 counts) (Figure 1(a)). The same DNA hypermethylation pattern is observed analyzed by Pathway analysis (Figure 1(b)).

3.3. Karyotype Status after Treatment. Certain amount of blood arsenic was observed in most patients $(7.6 \mathrm{ug} / \mathrm{L}$ to $35.4 \mathrm{ug} / \mathrm{L}$ ) after treatment. Hematologic responses were acquired after treatment with arsenic-containing Chinese herbal formula in 14 patients that have completed treatment for 6 months, including $2 \mathrm{CR}$ and 12 hematologic improvements. Of these 14 patients, no karyotype change was observed after treatment in most cases, with the exception of one case, whose karyotype changed from 46, XX [20], trisomy +8 signal $<1.0 \%$ (by FISH) before treatment, to 47 , $\mathrm{XX},+8[6],-\mathrm{X}[2] / 46, \mathrm{XX}[23]$, trisomy +8 signal $>40 \%$ (by
FISH) after treatment. We concluded that arsenic-containing Chinese herbal formula had no obvious effects on karyotype status.

\subsection{Demethylation Effects of Arsenic-Containing Chinese} Herbal Formula. Six months of treatment with arseniccontaining Chinese herbal formula significantly decreased number of methylated genes and their functional signaling pathways in MDS patients.

According to GO analysis tool, the number of hypermethylated gene decreased from 1063 to 75 after treatment. The functional pathways that involved gene hypermethylated decreased from 156 to 18 after treatment (Table 4). Further analysis indicated that the methylated genes involved in multicellular organismal development, signal transduction and apoptosis were demethylated after treatment.

Analyzed by Pathway analysis tool, the number of hypermethylated gene decreased from 318 to 21 after treatment. The involved functional pathways of the hypermethylated genes decreased from 47 to 8 (Table 5). The methylated genes involved in cancer signals, chemokine signaling pathway, MAPK signaling pathway, calcium signaling, and so forth, were demethylated after treatment.

Compared with multiple reports [16-23] and online database (http://www.ncbi.nlm.nih.gov/gene), our results indicate that 13 hypermethylated genes were demethylated after treatment (Table 6). Of these 13 demethylated genes, WNT8B, DCC, DACT1 involve multicellular organismal development; E2F3, NCOA4, HDAC1, PIAS3, ATF3, ZBTB33, LHX9 mainly involve transcription control; FASLG, TRAF3 involve cell apoptosis; PIK3R2 mainly involves signaling transduction.

Overall, our results indicated that after treatment with arsenic-containing Chinese herbal formula, the numbers of hypermethylated genes and the involved functional pathways were significantly decreased. The methylated genes involved 
TABLE 4: Functions of genes with hypermethylation after treatment.

\begin{tabular}{|c|c|c|c|c|}
\hline Go_id & Go_name & Diffgene_count & $P$ value & FDR \\
\hline GO:0007596 & Blood coagulation & 13 & $2.09 E-04$ & $2.11 E-02$ \\
\hline GO:0007088 & regulation of mitosis & 3 & $2.36 E-04$ & $2.11 E-02$ \\
\hline GO:0016337 & Cell-cell adhesion & 5 & $5.61 E-04$ & $2.55 E-02$ \\
\hline GO:0006357 & Regulation of transcription from RNA polymerase II promoter & 8 & $5.72 E-04$ & $2.56 E-02$ \\
\hline GO:0006325 & Chromatin organization & 3 & $1.11 E-03$ & $3.05 E-02$ \\
\hline GO:0006890 & Retrograde vesicle-mediated transport, Golgi to ER & 3 & $1.54 E-03$ & $3.22 E-02$ \\
\hline GO:0032312 & Regulation of ARF GTPase activity & 2 & $1.64 E-03$ & $3.25 E-02$ \\
\hline GO:0033690 & Positive regulation of osteoblast proliferation & 2 & $2.44 E-03$ & $3.79 E-02$ \\
\hline GO:0060710 & Chorioallantoic fusion & 2 & $2.44 E-03$ & $3.79 E-02$ \\
\hline GO:0006364 & rRNA processing & 4 & $3.02 E-03$ & $4.05 E-02$ \\
\hline GO:0043069 & Negative regulation of programmed cell death & 2 & $3.39 E-03$ & $4.19 E-02$ \\
\hline GO:0015031 & Protein transport & 9 & $3.50 E-03$ & $4.22 E-02$ \\
\hline GO:0005975 & Carbohydrate metabolic process & 8 & $4.92 E-03$ & $4.56 E-02$ \\
\hline GO:0006355 & Regulation of transcription, DNA-dependent & 20 & $5.43 E-03$ & $4.65 E-02$ \\
\hline GO:0007155 & Cell adhesion & 11 & $5.45 E-03$ & $4.66 E-02$ \\
\hline GO:0042953 & Lipoprotein transport & 2 & $5.75 E-03$ & $4.70 E-02$ \\
\hline GO:0048863 & Stem cell differentiation & 2 & $5.75 E-03$ & $4.70 E-02$ \\
\hline GO:0009395 & Phospholipid catabolic process & 2 & $7.14 E-03$ & $4.87 E-02$ \\
\hline
\end{tabular}

This table shows functions of genes with hypermethylation after treatment. go_id: gene ID number in Gene Ontology database; go_name: category of function of gene; diffgene_count: number of gene methylated; $P$ value: $P<0.01$ indicating methylation is significant; FDR: false discovery rate, $P<0.05$ as filter cutoff rate.

TABLE 5: Pathways of hypermethylated genes after treatment.

\begin{tabular}{lccc}
\hline Path_id & Path_name & diffgene_count & $P$ value \\
\hline 10 & Glycolysis / Gluconeogenesis & 4 & $5.97 E-03$ \\
4622 & RIG-I-like receptor signaling pathway & 4 & $8.21 E-03$ \\
5164 & Influenza A & 6 & $1.09 E-02$ \\
5215 & Prostate cancer & 4 & $3.82 E-02$ \\
4666 & Fc gamma R-mediated phagocytosis & 4 & $3.82 E-02$ \\
5131 & Shigellosis & 3 & $4.82 E-02$ \\
4964 & Proximal tubule bicarbonate reclamation & 2 & $3.35 E-02$ \\
5120 & Epithelial cell signaling in Helicobacter pylori infection & 3 & $3.68 E-02$ \\
\hline
\end{tabular}

This table shows pathways of hypermethylated genes after treatment. Path_id: gene ID number in KEGG database; Path_name: pathway of gene; diffgene_count: number of gene methylated in specific pathway; $P$ value: $P<0.01$ indicating methylation is significant; FDR: false discovery rate, $P<0.05$ as filter cutoff rate.

TABLe 6: Demethylated genes profiles after treatment.

\begin{tabular}{|c|c|c|c|c|}
\hline Go_id & Go_name & gene_symbol & $P$ value & FDR \\
\hline \multirow{3}{*}{ GO:0007275 } & \multirow{3}{*}{ Multicellular organismal development } & WNT8B & $1.05 E-09$ & $2.69 E-07$ \\
\hline & & DCC & $1.05 E-09$ & $2.69 E-07$ \\
\hline & & DACT1 & $1.05 E-09$ & $2.69 E-07$ \\
\hline \multirow{3}{*}{ GO:0045893 } & \multirow{3}{*}{ Positive regulation of transcription, DNA-dependent } & $\mathrm{E} 2 \mathrm{~F} 3$ & $1.12 E-07$ & $1.15 E-05$ \\
\hline & & NCOA4 & $1.12 E-07$ & $1.15 E-05$ \\
\hline & & HDAC1 & $1.12 E-07$ & $1.15 E-05$ \\
\hline \multirow{3}{*}{ GO:0006355 } & \multirow{3}{*}{ Regulation of transcription, DNA-dependent } & PIAS3 & $3.24 E-06$ & $2.08 E-04$ \\
\hline & & ATF3 & $3.24 E-06$ & $2.08 E-04$ \\
\hline & & ZBTB33 & $3.24 E-06$ & $2.08 E-04$ \\
\hline GO:0006915 & Apoptosis & FASLG & $2.10 E-05$ & $9.76 E-04$ \\
\hline GO:0045892 & Negative regulation of transcription, DNA-dependent & LHX9 & $2.32 E-05$ & $1.04 E-03$ \\
\hline GO:0006917 & Induction of apoptosis & TRAF3 & $6.13 E-05$ & $2.12 E-03$ \\
\hline GO:0007165 & Signal transduction & PIK3R2 & $2.58 E-04$ & $6.45 E-03$ \\
\hline
\end{tabular}


in MDS pathogenesis were de-methylated after treatment with arsenic-containing herbal formula.

\section{Discussion}

Aberrant DNA methylation is frequently observed in the myeloid malignancies, including myelodysplastic syndrome (MDS). With most researches focusing on single gene methylation [17-23], the limitations of these researches are obvious regarding global DNA methylation in MDS. In this study, using global DNA methylation method (ChIP-on-chip assays), we investigated genomic wide DNA methylation level in MDS thus reveal overall methylation profiling in MDS. We also determined the effect of arsenic-containing Chinese herbal formula on karyotype and the genomic methylation level in MDS patients.

Our results indicated that among 4 million genes being tested 1063 genes were hypermethylated in MDS patients compared with control. The hypermethylated genes involve 156 functional pathways. Currently over 30 hypermethylated genes have been identified in MDS patients [16-23]. Confirmatively, we observed 17 of these hypermethylated genes in this study, namely FZD1, SOCS2, DKK1, DACT1, GADD45B, GADD45G, ARHGAP8, ARHGAP44, CDH7, ZBTB32, ZBTB33, ZBTB40, PAX2, ATF3, POU4F2, LHX2, and LHX9. The function of these genes is highly related to the pathogenesis of MDS.

After analyzing the correlation of DNA methylation status and karyotypes, we found that the number of genes with aberrant methylation is the highest in MDS patients with normal karyotype, higher in trisomy 8 karyotype, and relative low in patients with cytogenetic abnormalities other than trisomy 8 karyotype. Interestingly, we found that after treatment with arsenic-containing Chinese herbal formula, clinical outcome of MDS patients with normal karyotype or trisomy 8 is better than that of the patients with other cytogenetic abnormalities [4]. However arsenic-containing Chinese herbal formula had no effects on karyotype status of the patients.

Furthermore, after treatment with arsenic-containing Chinese herbal formula, the number of hypermethylated genes decreased from 1063 to 75 ; the functional pathways affected by these hypermethylated genes decreased from 156 to 18 . The methylated genes involved in multicellular organismal development, signal transduction, and apoptosis were demethylated after treatment. We identified 13 genes, whose function involving in multicellular organismal development, transcription control, signal transduction, apoptosis, cell development, and adhesion, as well as pathways in cancer, had been completely demethylated after treatment. The identified 13 demethylated genes including WNT8B, DCC, DACT1, E2F3, NCOA4, HDAC1, PIAS3, ATF3, ZBTB33, LHX9, FASLG, TRAF3, and PIK3R2), are critical for pathogenesis of MDS. Our research uncovered a novel DNA demethylating activity of Realgar-containing Chinese herbal formula in the treatment of MDS.

Currently DNA hypomethylating agents like the cytidine nucleoside analogs azacitidine and decitabine are widely used for the treatment of patients with myelodysplastic syndromes. Both drugs effected DNA methyltransferase1 depletion and DNA hypomethylation [24-27]. However based on detoxification process of arsenic, we reason that arsenic hypomethylates DNA in a different pathway from azacitidine. Methylation of arsenic had been recognized as a detoxification process through methyltransferase enzymes using S-adenosylmethionine as methyl donor. Therefore the observed hypomethylating effects of arsenic in this study might be due to its competitive exhaustion of methyl during detoxification process of arsenic. Details regarding this mechanism are currently under investigation in the lab.

In summary, in this study we found that aberrant DNA methylation correlates with MDS pathogenesis; the number of aberrant DNA methylation is highest in patients with normal karyotype, followed by trisomy 8 karyotype and relative low in patients with other cytogenetic abnormalities; treatment with arsenic-containing Chinese herbal formula resulted in a significant genome-wide demethylation, with no effects on karyotype status; the function of the demethylated genes is largely involved in transcription control, signal transduction, cell apoptosis, and multicellular organismal development. Our research uncovered a novel DNA demethylating activity of arsenic-containing Chinese herbal formula in the treatment of MDS.

\section{Conflict of Interests}

The authors have no conflict of interests to declare.

\section{Acknowledgments}

The authors thank Dr. Wang, Zhanxiang from Indiana University School of Medicine, United States, for critical suggestions in preparing this paper. This study was supported by a Grant from the National Natural Science Foundation of China (no. 30973812 ).

\section{References}

[1] Y. Jiang, A. Dunbar, L. P. Gondek et al., "Aberrant DNA methylation is a dominant mechanism in MDS progression to AML," Blood, vol. 113, no. 6, pp. 1315-1325, 2009.

[2] S. Xu, R. Ma, X. M. Hu, Y. G. Xu, X. H. Yang, H. Z. Wang et al., "Clinical observation on qinghuang powder for treatment of 31 cases of myelodysplastic syndrome," Zhong Yi Za Zhi, vol. 47, no. 7, pp. 514-516, 2006.

[3] S. Xu, X. M. Hu, and Y. G. Xu, "Effect of treatment for myelodysplastic syndrome by Qinghuang Powder combined with Chinese herbs for reinforcing shen and strenghening pi," Zhong Guo Zhong Xi Yi Jie He Za Zhi, vol. 28, no. 3, pp. 216218, 2008.

[4] S. Xu, R. Ma, X. M. Hu, Y. G. Xu, X. H. Yang, H. Z. Wang et al., "Clinical observation of the treatment of myelodysplastic syndrome mainly with qinghuang powder," Chinese Journal of Integrative Medicine, vol. 17, no. 11, pp. 834-839, 2011.

[5] J. W. Vardiman, J. Thiele, D. A. Arber et al., "The 2008 revision of the World Health Organization (WHO) classification of myeloid neoplasms and acute leukemia: rationale and important changes," Blood, vol. 114, no. 5, pp. 937-951, 2009. 
[6] B. D. Cheson, J. M. Bennett, H. Kantarjian et al., "Report of an international working group to standardize response criteria for myelodysplastic syndromes," Blood, vol. 96, no. 12, pp. 3671-3674, 2000.

[7] A. Tefferi, G. Barosi, R. A. Mesa et al., "International Working Group (IWG) consensus criteria for treatment response in myelofibrosis with myeloid metaplasia, for the IWG for Myelofibrosis Research and Treatment (IWG-MRT)," Blood, vol. 108, no. 5, pp. 1497-1503, 2006.

[8] F. Mitelman, ISCN: An International System for Human Cytogenetic Nomenclature, S. Karger, Basel, Swizeland, 1995.

[9] T. Z. Berardini, "The Gene Ontology in 2010: extensions and refinements," Nucleic Acids Research, vol. 38, no. 1, Article ID gkp1018, pp. D331-D335, 2009.

[10] M. Ashburner, C. A. Ball, J. A. Blake et al., "Gene ontology: tool for the unification of biology," Nature Genetics, vol. 25, no. 1, pp. 25-29, 2000.

[11] K. F. Aoki-Kinoshita and M. Kanehisa, "Gene annotation and pathway mapping in KEGG," Methods in Molecular Biology, vol. 396, pp. 71-91, 2007.

[12] M. Kanehisa and Subramaniam, "The KEGG database," Novartis Foundation Symposium, vol. 247, pp. 91-103, 2002.

[13] M. Abe, M. Ohira, A. Kaneda et al., "CpG island methylator phenotype is a strong determinant of poor prognosis in neuroblastomas," Cancer Research, vol. 65, no. 3, pp. 828-834, 2005.

[14] E. L. Greer and Y. Shi, "Histone methylation: a dynamic mark in health, disease and inheritance," Nature Reviews Genetics, vol. 13, no. 5, pp. 343-357, 2012.

[15] S. Kouidou, A. Malousi, and N. Maglaveras, "Methylation and repeats in silent and nonsense mutations of p53," Mutation Research, vol. 599, no. 1-2, pp. 167-177, 2006.

[16] X. U. Feng and L. I. Xiao, "Epigenetic alterations in myelodysplastic syndromes," Journal of Experimental Hematology, vol. 18, no. 2, pp. 531-535, 2010.

[17] Y. Zhao, J. Bo, L. P. Dou et al., "Preliminary study on difference of Id4 gene methylation in various types of myelodysplastic syndromes," Zhongguo Shi Yan Xue Ye Xue Za Zhi, vol. 17, no. 3, pp. 618-620, 2009.

[18] L. Yu, C. Liu, J. Vandeusen et al., "Global assessment of promoter methylation in a mouse model of cancer identifies ID4 as a putative tumor-suppressor gene in human leukemia," Nature Genetics, vol. 37, no. 3, pp. 265-274, 2005.

[19] Y. Jiang, A. Dunbar, L. P. Gondek et al., "Aberrant DNA methylation is a dominant mechanism in MDS progression to AML," Blood, vol. 113, no. 6, pp. 1315-1325, 2009.

[20] R. Claus, B. Hackanson, A. R. Poetsch, M. Zucknick, M. Sonnet, N. Blagitko-Dorfs et al., "Quantitative analyses of DAPK1 methylation in AML and MDS," International Journal of Cancer, vol. 131, no. 2, pp. E138-E142, 2012.

[21] S. J. Wu, M. Yao, W. C. Chou, J. L. Tang, C. Y. Chen, B. S. Ko et al., "Clinical implications of SOCS1 ethylation in myelodysplastic syndrome," British Journal of Haematology, vol. 135, no. 3, pp. 317-323, 2006.

[22] J. Lin, D. M. Yao, J. Qian et al., "Methylation status of fragile histidine triad (FHIT) gene and its clinical impact on prognosis of patients with myelodysplastic syndrome," Leukemia Research, vol. 32, no. 10, pp. 1541-1545, 2008.

[23] H. Y. Kang, C. Wang, L. P. Dou et al., "Methylation status of ZO-1 gene in patients with myelodysplastic syndrome," Journal of Experimental Hematology, vol. 16, no. 1, pp. 70-73, 2008.

[24] G. Egger, G. Liang, A. Aparicio, and P. A. Jones, "Epigenetics in human disease and prospects for epigenetic therapy," Nature, vol. 429, no. 6990, pp. 457-463, 2004.
[25] J. P. F. Issa, V. Gharibyan, J. Cortes et al., "Phase II study of low-dose decitabine in patients with chronic myelogenous leukemia resistant to imatinib mesylate," Journal of Clinical Oncology, vol. 23, no. 17, pp. 3948-3956, 2005.

[26] J. P. J. Issa, H. M. Kantarjian, and P. Kirkpatrick, "Azacitidine," Nature Reviews Drug Discovery, vol. 4, no. 4, pp. 275-276, 2005.

[27] H. Kantarjian, Y. Oki, G. Garcia-Manero et al., "Results of a randomized study of 3 schedules of low-dose decitabine in higher-risk myelodysplastic syndrome and chronic myelomonocytic leukemia," Blood, vol. 109, no. 1, pp. 52-57, 2007. 


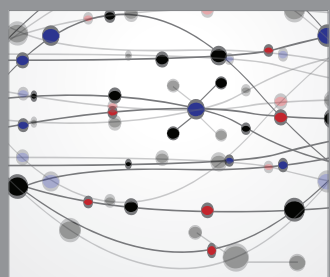

The Scientific World Journal
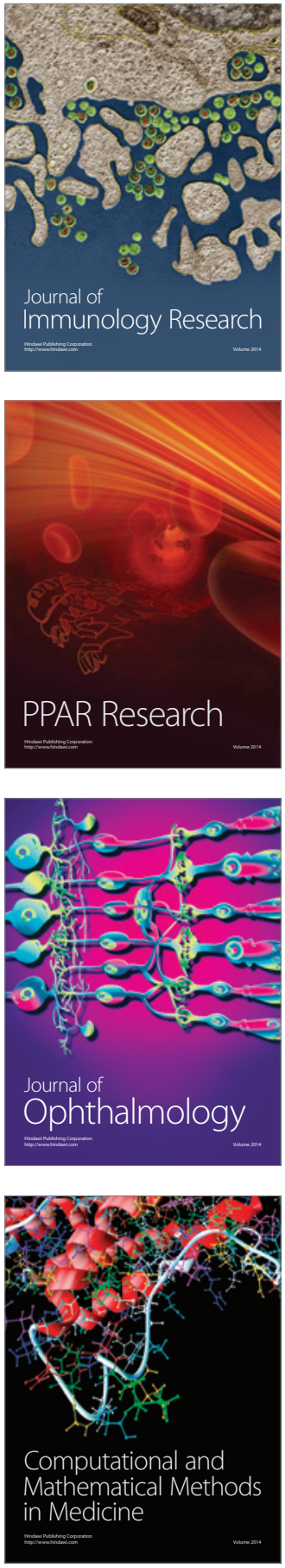

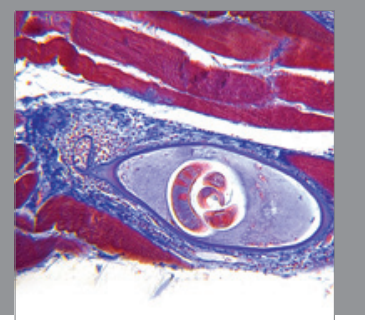

Gastroenterology

Research and Practice
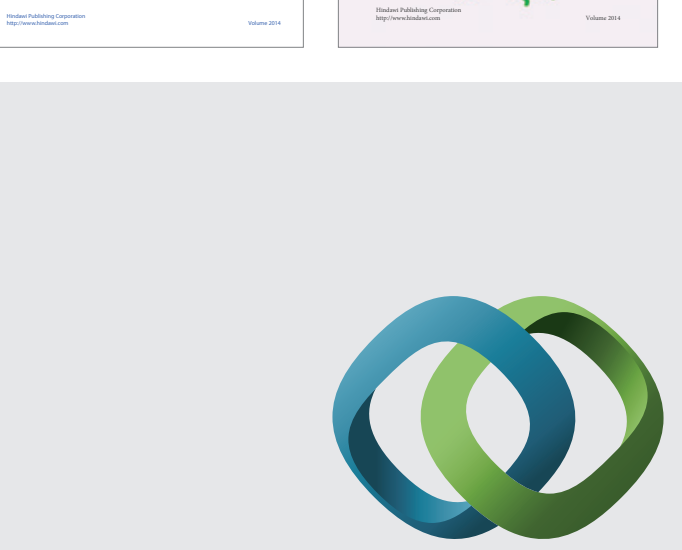

\section{Hindawi}

Submit your manuscripts at

http://www.hindawi.com
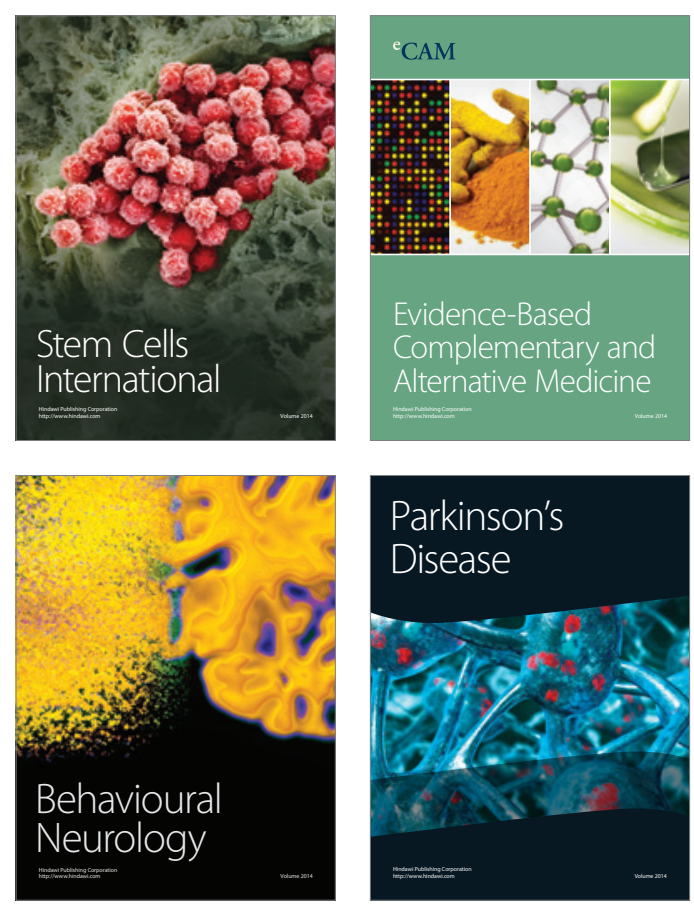

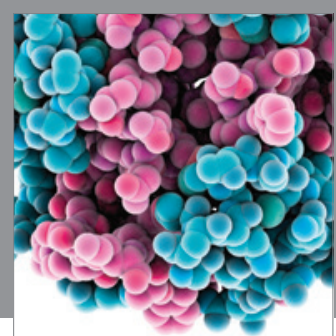

Journal of
Diabetes Research

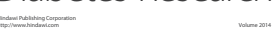

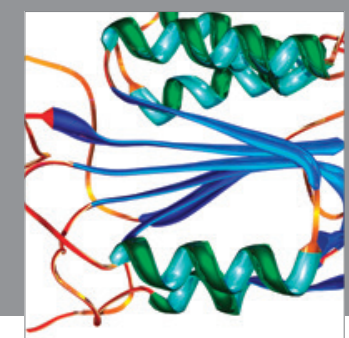

Disease Markers
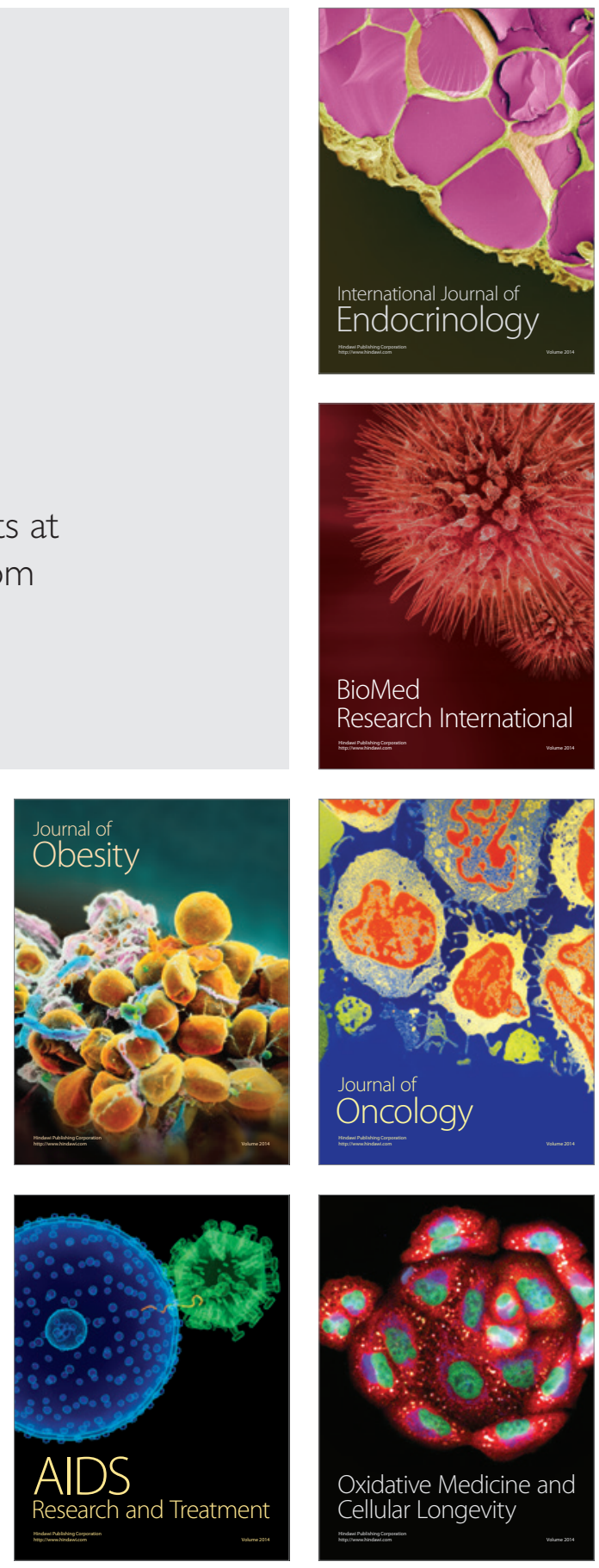